\title{
ATR suppresses the pro-tumorigenic functions of breast stromal fibroblasts
}

\author{
Mysoon M. Al-Ansari ${ }^{1,2}$ and Abdelilah Aboussekhra ${ }^{1}$ \\ ${ }^{1}$ Department of Molecular Oncology, King Faisal Specialist Hospital and Research Center, Riyadh, Saudi Arabia \\ ${ }^{2}$ Department of Microbiology, Faculty of Science and Medical Studies, King Saud University, Riyadh, Saudi Arabia \\ Correspondence to: Abdelilah Aboussekhra, email: aboussekhra@kfshrc.edu.sa \\ Keywords: ATR; breast cancer; cancer-associated fibroblasts; p53 \\ Received: July 23, $2018 \quad$ Accepted: September 09, $2018 \quad$ Published: October 05, 2018 \\ Copyright: Al-Ansari et al. This is an open-access article distributed under the terms of the Creative Commons Attribution License \\ 3.0 (CC BY 3.0), which permits unrestricted use, distribution, and reproduction in any medium, provided the original author and \\ source are credited.
}

\section{ABSTRACT}

The ATR protein kinase is a master regulator of the cellular responses to DNA damage and replication stresses. Despite these crucial physiological roles, the implication of ATR in human carcinogenesis remains elusive. We have shown here that the ATR level is reduced in most cancer-associated fibroblasts (CAFs) as compared to their adjacent normal counterparts. Importantly, specific ATR knockdown activated breast fibroblasts, and enhanced their paracrine pro-carcinogenic effects via strong increase in the expression/secretion of SDF-1 and IL-6. Furthermore, ATR-deficient fibroblasts enhanced tumor growth and aggressiveness in orthotopic breast tumor xenografts. On the other hand, ectopic expression of ATR suppressed the expression/ secretion of several cancer-promoting proteins such as IL-6, TGF- $\beta 1$ and SDF-1, and inhibited the migration and invasion capacities of breast myofibroblast cells. Furthermore, ATR up-regulation in active breast fibroblasts reduced their paracrine pro-migratory/-invasive effects on breast cancer cells. Interestingly, the cancer promoting effects of ATR-deficient cells were repressed by ectopic expression of the ATR effector p53. These results indicate that ATR is a major target of cancer cells in breast fibroblasts wherein this protein kinase represses both autocrine and paracrine pro-carcinogenic effects. This indicates that the ATR status in these cells could be of great prognostic/diagnostic values.

\section{INTRODUCTION}

Several lines of evidence indicate that cancerassociated fibroblasts (CAFs), which constitute the major component of the tumor stroma, actively participate in breast tumor growth and spread $[1,2]$. Active fibroblasts or myofibroblasts promote tumor growth through secreting several soluble pro-carcinogenic factors such as SDF-1 and IL-6 $[3,4]$. The expression of these factors is repressed by several tumor suppressor proteins, such as p16 and p53, which are cell cycle checkpoint proteins with non-cell-autonomous tumor suppressive function as well $[5,6]$. Activation of the p53 pathway occurs in response to cellular stresses, which appear to be dependent on distinct upstream regulatory kinases. One of these important protein kinases is the Ataxia-Telangiectasia and Rad-3 related (ATR) protein. The ATR protein kinase is a cell cycle checkpoint protein, which coordinates cellular responses to DNA damage and replication stress, and therefore it is essential for the maintenance of genomic integrity [7]. The ATR gene is essential, and its disruption leads to early embryonic lethality in mice and formation of large benign tumors in heterozygote animals $[8,9]$. However, hypomorphic mutations in the gene, with only partial loss of function, lead to the rare hereditary disorder Seckel syndrome [10, 11]. Based on several studies, ATR is not mutated in the vast majority of human cancers, including breast and ovarian carcinomas [12]. However, changes in the expression level of this protein kinase could play a major role in carcinogenesis. 
In this study, we addressed the role of ATR in regulating the activity of breast stromal fibroblasts and their paracrine procarcinogenic effects. We have clearly shown that ATR has non-cell-autonomous tumor suppressive functions both in vitro and in animal models.

\section{RESULTS}

\section{ATR is down-regulated in active breast cancer- associated fibroblasts}

We started the present study by assessing the ATR expression level in 12 human breast CAFs and their counterpart fibroblasts isolated from histologically normal adjacent cancer-free tissues (TCFs). CAF/TCF pairs were always used simultaneously at similar passages. Whole cell extracts were prepared and specific anti-ATR and antiGAPDH (used as internal control) antibodies were utilized for immunoblotting analysis. Figure $1 \mathrm{~A}$ and $1 \mathrm{~B}$ shows that the ATR level is lower in 9 out of 12 CAFs (75\%) as compared to their corresponding TCFs. However, ATR level was similar in $3 \mathrm{CAF} / \mathrm{TCF}$ pairs, namely 69,118 and 180 (Figure 1A-1B). Furthermore, a great inter-individual variation in ATR expression was observed between the various CAFs and also TCFs (Figure 1A-1B).

\section{ATR down-regulation activates breast stromal fibroblasts}

To evaluate the autocrine and the paracrine effects of ATR down-regulation in breast stromal fibroblasts, we knocked-down ATR using specific shRNA in TCF-64 cells, while a scrambled sequence was used as control. Figure 2A shows that the ATR protein level was reduced in the ATR-shRNA-treated cells (N64-sh) as compared to their corresponding control cells (N64C). This decrease was accompanied with a strong increase in the levels of $\alpha$-SMA, SDF-1, IL- 6 and TGF- $\beta 1$ (four important markers of active stromal fibroblasts) (Figure 2A). Similar results were obtained using another ATR-shRNA sequence (N64-sh2) (Figure 2B). Likewise, ATR downregulation increased the expression of MMP-2 (Figure 2A). However, ATR down-regulation reduced the level of the tumor suppressor protein p53 and its target p21 (Figure 2A). Moreover, ATR deficiency activated Jak2 and STAT3, which led to the up-regulation of their downstream target AUF1 (Figure 2A). These results suggest that ATR represses breast stromal fibroblasts through inhibition of the Jak2/STAT3 pathway.

Figure 2C shows that the mRNA levels of the $T G F-\beta 1, S D F-1, \alpha-S M A, I L-6$ and $M M P-2$ genes were strongly increased in ATR-defective cells as compared to their corresponding control cells. Similar results were obtained using ATR-shRNA2 in TCF-64 (Figure 2D). These genes were also up-regulated in ATR-deficient cells (N64-sh) as compared to controls (N64C), when these cells were grown in 3D culture conditions (Figure 2E).

We have next assessed the effect of ATR downregulation on the secretion of some important cytokines. Therefore, N64-sh and N64C cells were cultured in SFM for $24 \mathrm{~h}$, and the resulting SFCM were collected and the levels of the secreted proteins were assessed by ELISA.
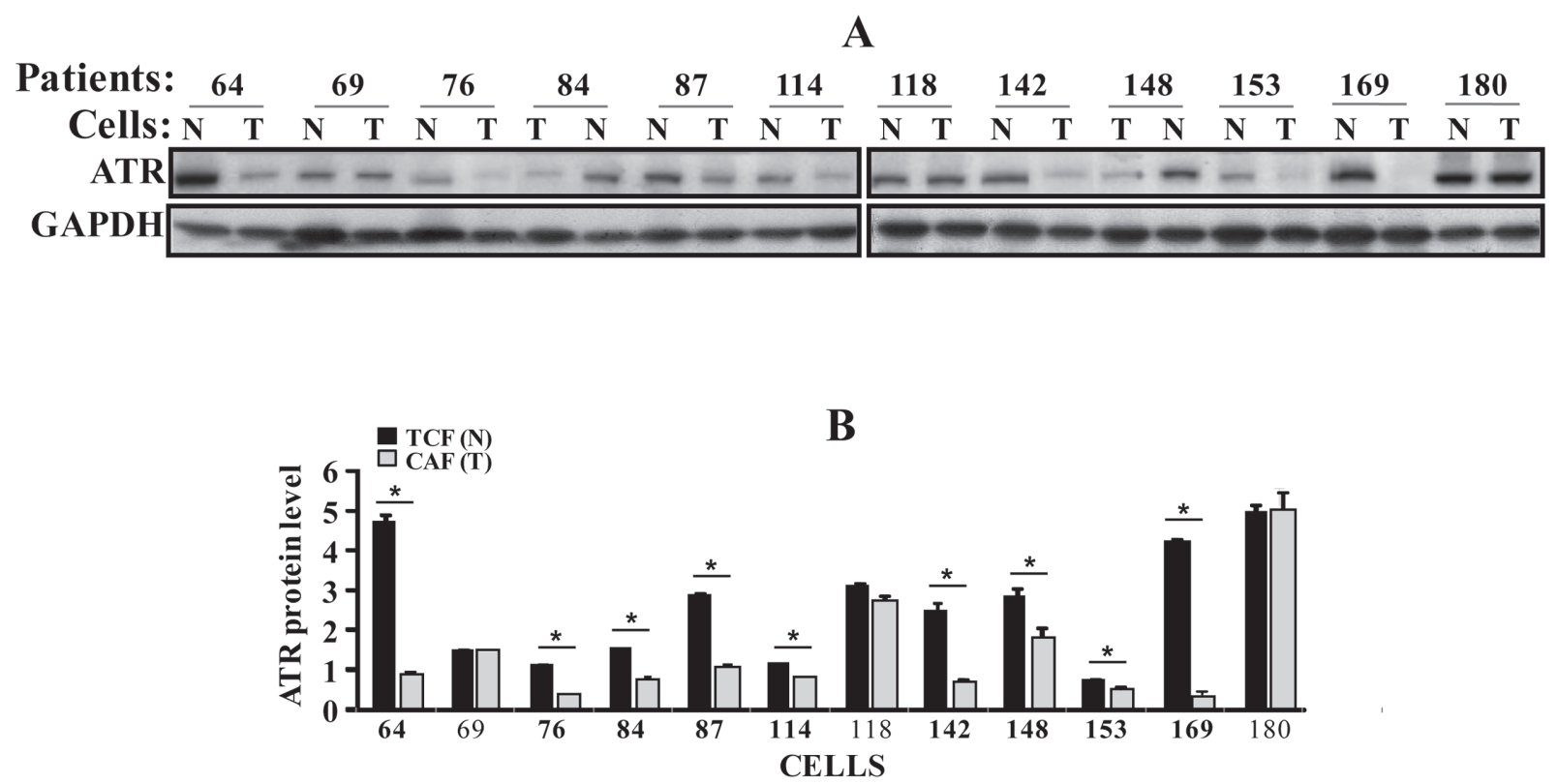

Figure 1: ATR is down-regulated in CAFs. (A) Whole cell lysates were prepared from the indicated CAF (T) and TCF (N) cells and $50 \mu \mathrm{g}$ of proteins were used for immunoblotting analysis using antibodies against the indicated proteins. (B) Histogram, the values were determined by densitometry following immunoblotting and normalized against GAPDH. Error bars indicate mean \pm SD for 3 different experiments, ${ }^{*} P<0.001$. 
Figure 2F shows that the secreted levels of the MMP-2, SDF-1, IL-6 and TGF- $\beta 1$ proteins significantly increased in N64-sh cells as compared to N64C. Similar results were obtained when ATR was knocked-down using ATRshRNA1 in normal breast fibroblast cells (NBF-1) (Figure $2 \mathrm{G})$. This indicates that ATR negatively controls the expression/secretion of these cancer-promoting proteins in breast stromal fibroblasts.

We have next investigated the effect of ATR downregulation on their invasion and migration abilities. Figure $2 \mathrm{H}$ shows that the number of migrating and invading ATRdeficient cells (N64-sh) was significantly higher than the number of migrating and invading control cells (N64C). This indicates that ATR represses both the migratory and the invasiveness abilities of breast stromal fibroblasts.

\section{ATR inhibits the paracrine pro-carcinogenic effects of breast stromal fibroblasts through suppressing the expression/secretion of SDF-1 and IL-6}

In addition, we have utilized SFCM from N64-sh cells (N64-sh-SFCM) and N64C cells (N64C-SFCM) to treat MCF-7 cells in 96-well plates. The real-time cell
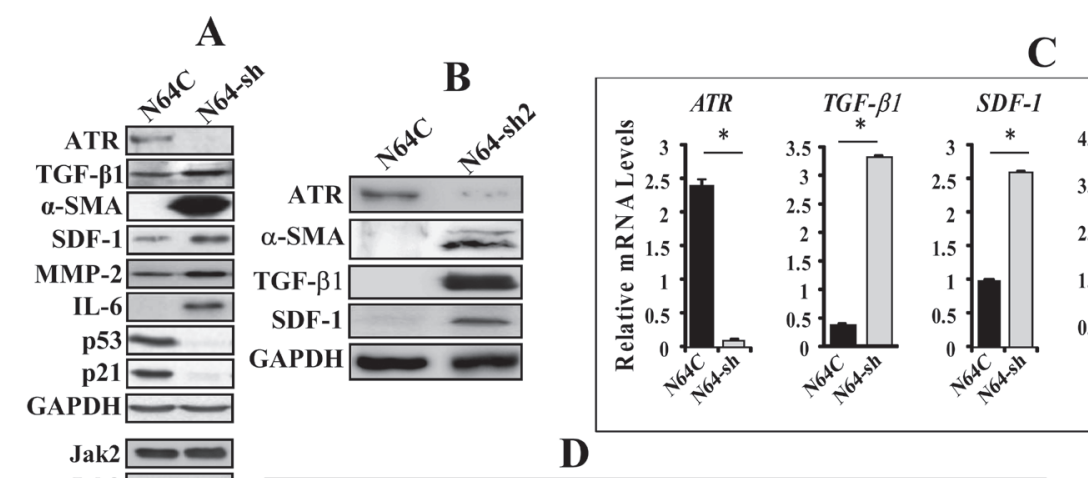

C
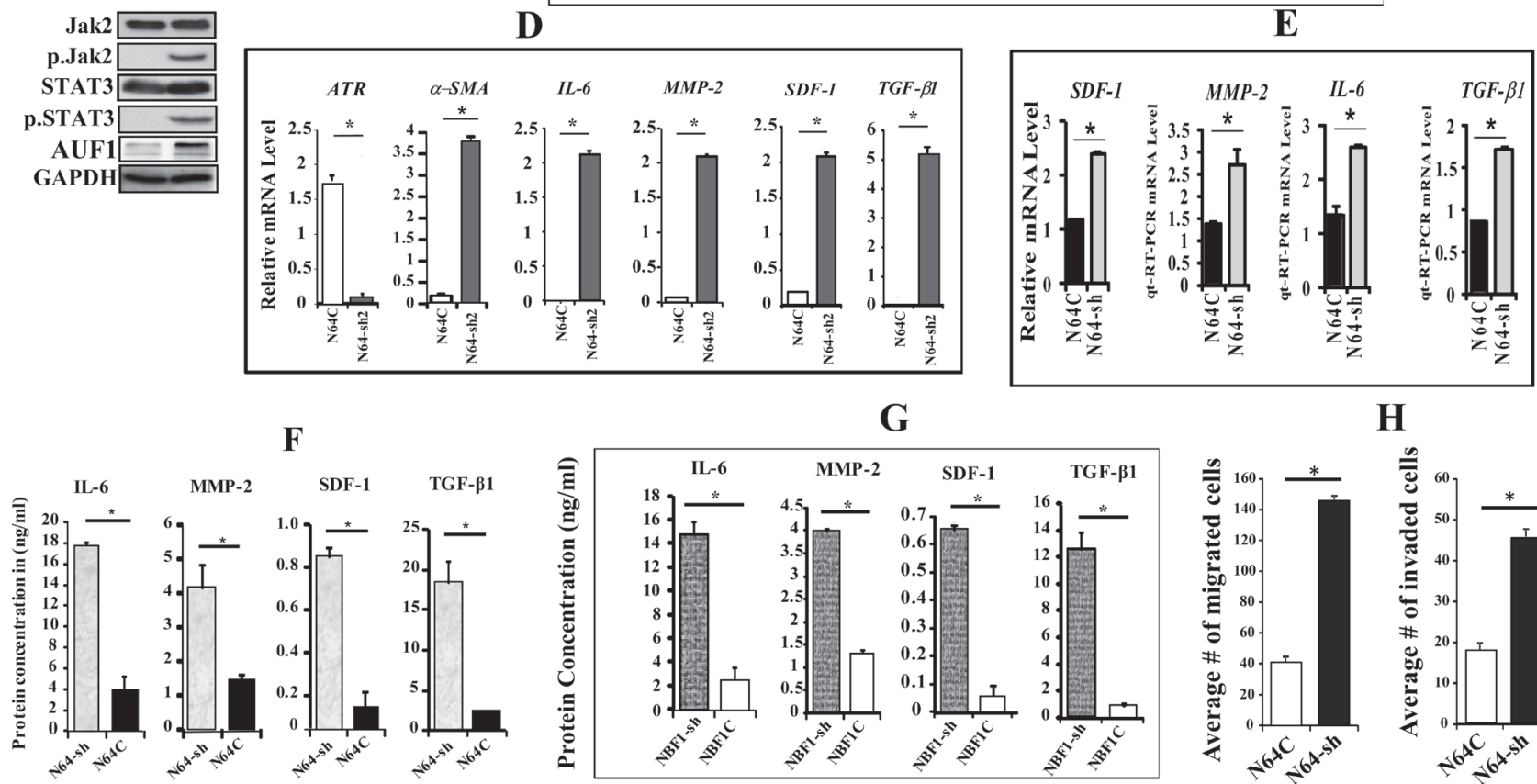

G

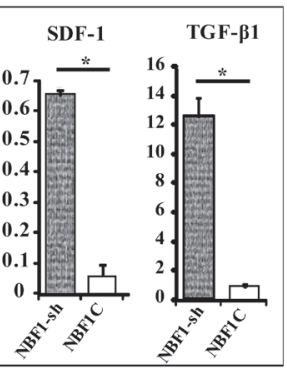

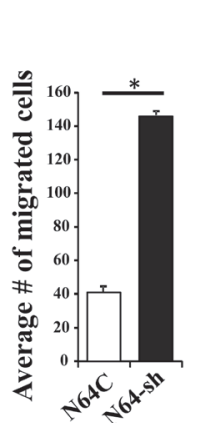

$\mathbf{H}$

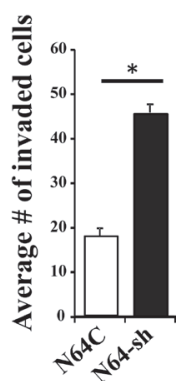

Figure 2: ATR down-regulation activates breast stromal fibroblasts. TCF-64 cells were transfected with specific ATRshRNA (N64-sh) or a scrambled sequence (N64C). (A and B) Whole cell lysates were prepared from the indicated cells and were used for immunoblotting analysis using antibodies against the indicated proteins. (C) Total RNA was extracted from the indicated cells, and the mRNA levels of the indicated genes were assessed by qRT-PCR using specific primers. Error bars represent mean $\pm \mathrm{S} . \mathrm{D}$. ${ }^{*} P<4 \mathrm{x} 10^{-6}$. (D) Total RNA was extracted from the indicated cells, and the mRNA levels of the indicated genes were assessed by qRT-PCR using specific primers. Error bars represent mean \pm S.D. ${ }^{*} P<4 \times 10^{-6}$. (E) N64-sh and N64C cells were seeded separately over 3D networks Biotech in 24 well plate, and then were allowed to grow under observation. After 10 days, total RNA was extracted, and the mRNA levels of the indicated genes were assessed by qRT-PCR. Error bars represent mean \pm S.D. ${ }^{*} P<0.031$. (F and G) SFCM were collected from the indicated cells and the levels of the indicated proteins were determined by ELISA. Error bars represent mean \pm S.D ( $=3$ ). ${ }^{*} P<0.017811$ and ${ }^{*} P<0.003$, respectively. (H) Cells $\left(10^{5}\right)$ were seeded in SFM onto the upper compartment of the migration and invasion plates and incubated for $24 \mathrm{~h}$ in the presence of $\mathrm{CpM}$ in the lower chambers of 24-well BD BioCoat plates. The numbers of migrated/invaded cells were calculated and represented in histograms. Error bars represent mean $\pm \mathrm{SD}(\mathrm{n}=3) .{ }^{*} P<3.1 \times 10^{-5}$. 
electronic sensing system was used to monitor the effect of each SFCM on cellular proliferation. Figure 3A shows that N64-sh-SFCM enhanced the proliferation rate of MCF-7 cells more than 4 fold as compared to cells grown in the presence of N64C-SFCM. Similarly, the migration and invasion abilities of MCF-7 cells were 2.25 and 3.75 fold higher in the presence of N64-sh-SFCM than in the presence of N64C-SFCM, respectively (Figure 3B). Similar results were obtained with MDA-MB-231 cultured with SFCM derived from NBF1-sh1 or NBF1C (Figure 3C). This indicates that ATR-deficient breast stromal fibroblasts enhance the proliferation, migration and invasion abilities of breast cancer cells through paracrine secreted factors.

Subsequently, the level of the active forms of Erk1/2 and Akt were assessed by immunoblotting in MCF-7 cells treated with N64-sh-SFCM or N64C-SFCM. Figure 3D shows that while the levels of the inactive forms of these proteins did not change, the levels of the phosphorylated forms increased in MCF-7 cells treated with N64-sh-SFCM, as compared with their respective control. These factors are known to induce the motile and invasive capacities of cancer cells through the promotion of the epithelial-mesenchymal transition (EMT). To further confirm this transition, we assessed the expression levels of epithelial and mesenchymal markers in MCF-7 cells exposed to SFCM from N64-sh and N64C cells. N64-sh-SFCM decreased the level of EpCAM and E-cadherin, and up-regulated N-cadherin, vimentin, ZEB1 and Twist1 (important mesenchymal markers), as compared to control cells (Figure 3D). Together, these results indicate that ATR down-regulation in breast stromal fibroblasts triggers the EMT process in breast cancer cells in a paracrine manner, which demonstrates their active status.

Next, we sought to investigate the possible role of SDF-1 and IL-6 in these paracrine pro-invasive/
A

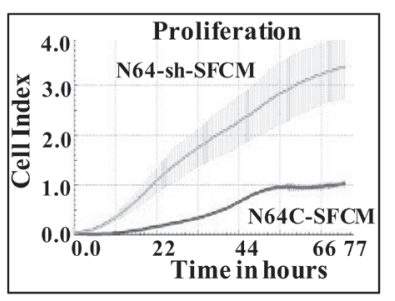

D

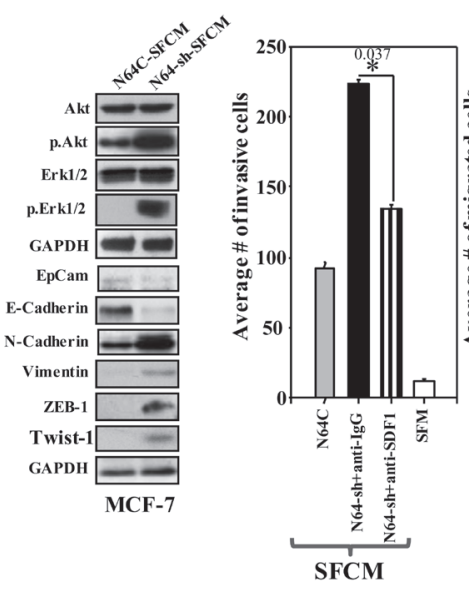

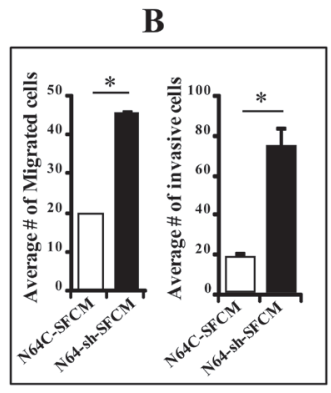

$\mathbf{E}$

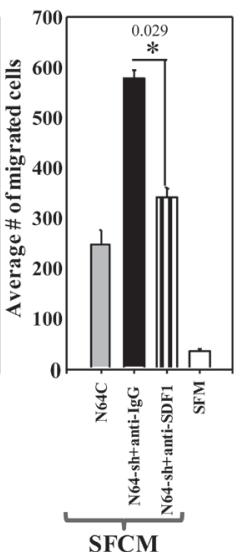

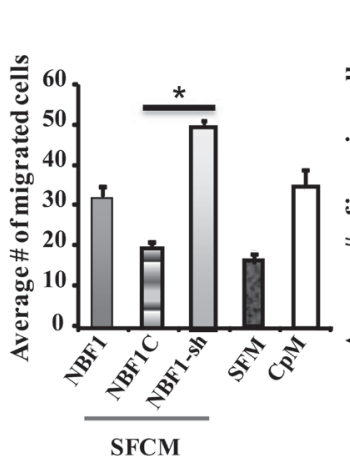

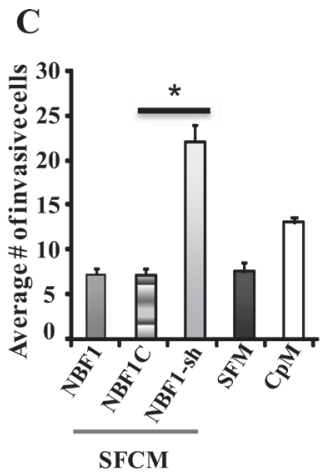

F
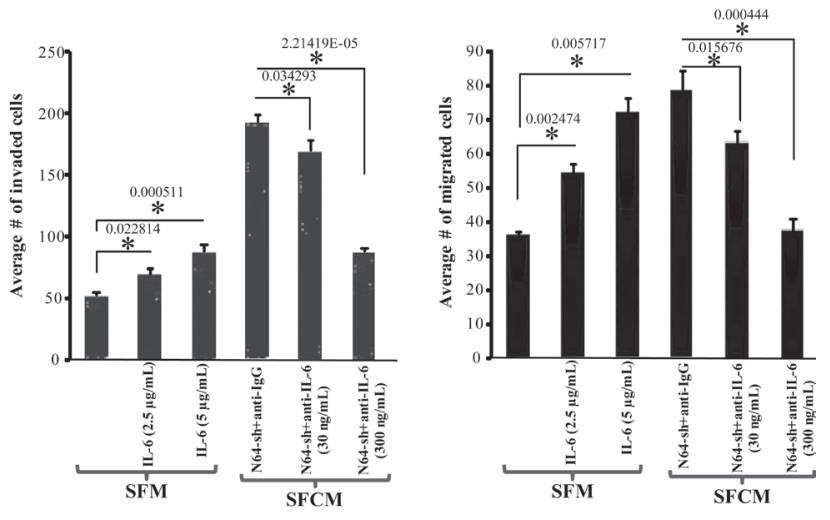

Figure 3: ATR down-regulation enhances the paracrine pro-carcinogenic effects of breast stromal fibroblasts in an SDF-1/IL-6-dependent manner. (A) SFCM collected from the indicated cells were added separately to MCF-7 cells previously seeded into 96 wells, and cell proliferation was assessed by the real-time cell electronic sensing system. (B) MCF-7 cells were seeded onto the upper compartment of the migration and invasion plates, and then were incubated for $24 \mathrm{~h}$ in the presence of SFCM from the indicated cells. Error bars represent mean \pm S.D $(n=3) .{ }^{*} P<0.0002$. (C) MDA-MB-231 cells were seeded onto the upper compartment of the migration and invasion plates, and then were incubated for $24 \mathrm{~h}$ in the presence of SFCM from the indicated cells. SFM and CpM were used as controls. Error bars represent mean \pm S.D $(n=3) .{ }^{*} P<1.7 \times 10^{-3}$. (D) Whole cell lysates were prepared from MCF-7 cells that were treated for $24 \mathrm{~h}$ with SFCM collected from the indicated cells. Immunoblotting analysis was performed using the indicated antibodies. (E and F) MCF-7 cells were seeded onto the upper compartment of the migration and invasion plates, and then were incubated for $24 \mathrm{~h}$ in the presence of the indicated media. Neutralizing antibodies (anti-SDF-1, anti-IL-6) or recombinant human IL-6 protein were separately added to SFCM or SFM, respectively. IgG was utilized as control. The numbers of migrated and invaded cells were represented in histograms. Error bars represent means $\pm \mathrm{S} . \mathrm{D}(\mathrm{n}=3) .{ }^{*} P$-values are indicated. 
migratory effects of ATR-deficient fibroblasts. To do this, N64-sh-SFCM was challenged either with anti-IgG antibody (used as negative control) or with anti-SDF-1 neutralizing antibody, and the effect on the migration/ invasion abilities of the MCF-7 cells were studied as described above. Figure 3E confirms the pro-migratory/ invasive effects of $\mathrm{N} 64 \mathrm{sh}-\mathrm{SFCM}$ relative to $\mathrm{N} 64 \mathrm{C}$ SFCM. However, the neutralizing anti-SDF-1 antibody repressed these paracrine effects (Figure 3E). We have subsequently inhibited IL-6 in N64-sh-SFCM using 2 different concentrations of neutralizing anti-IL-6 antibody (30 and $300 \mathrm{ng} / \mathrm{ml}$ ) for $24 \mathrm{~h}$, IgG was utilized as control. Figure $3 \mathrm{~F}$ shows that the inhibition of IL-6 significantly reduced the migration/invasion abilities of $\mathrm{MCF}-7$ cells in a concentration-dependent manner. On the other hand, addition of recombinant IL-6 to SFM enhanced the migration/invasion abilities of MCF-7 cells in a dosedependent manner (Figure 3F). These results indicate that SDF- 1 and IL- 6 are potent mediators of the paracrine promigratory/invasiveness capacities of ATR-deficient breast stromal fibroblasts.

\section{ATR-deficient fibroblasts stimulate breast cancer orthotopic tumor growth in mice}

To investigate the effect of ATR deficiency in human breast fibroblasts on tumor growth in vivo, 10 nude mice were randomized into two groups $(n=5)$ and orthotopic breast tumor xenografts were created under the nipples by co-implantation of MDA-MB-231 cells $\left(2 \times 10^{6}\right)$ with either N64-sh or N64C cells $\left(2 \times 10^{6}\right)$. All mice developed tumors. However, tumors bearing ATRdeficient fibroblasts (T-N64-sh) appeared earlier and grew vigorously faster relative to those having control cells (T-N64C) (Figure 4A). This indicates that ATRdeficient BSFs can enhance orthotopic tumor growth in mice.

We have next excised tumors and performed hematoxylin and eosin staining for histopathological analysis. Figure 4B shows abundant nuclei spindle shape in T-N64-sh as compared to T-N64C. In addition, T-N64-sh tissues showed no tubular structures, which were clearly developed in T-N64C tissues (Figure 4B). This shows that ATR-deficient BSFs enhance tumor aggressiveness.

In addition, Figure 4C shows that T-N64-sh tumors express higher level of the proliferation marker Ki-67, as well as cyclin D1 than the control tissue. Likewise, the levels of IL- 6 and TGF- $\beta 1$ were higher in T-N64-sh than in T-N64C tissues (Figure 4C). Furthermore, N64-sh cells activated STAT3 and Erk1/2 in tumor xenografts (Figure $4 C)$. Figure $4 \mathrm{C}$ shows also that while the levels of the mesenchymal markers N-cadherin, ZEB1, and vimentin were higher in the T-N64-sh tumors, EpCAM was downregulated in these tumors as compared to T-N64C. This shows that the presence of ATR-deficient breast stromal fibroblasts enhanced the EMT process in breast cancer cells in orthotopic tumor xenografts.
$\mathbf{A}$

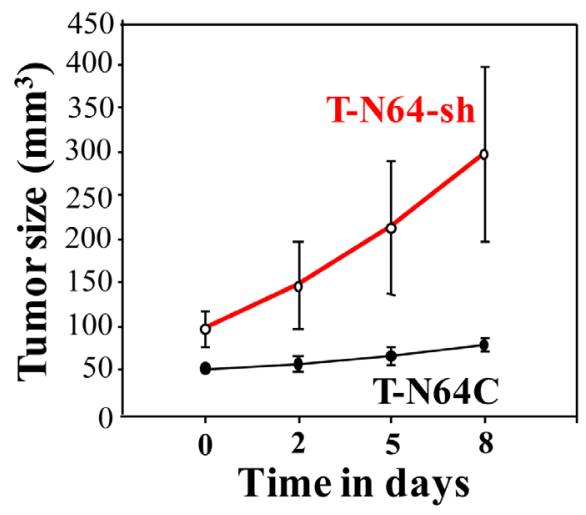

B

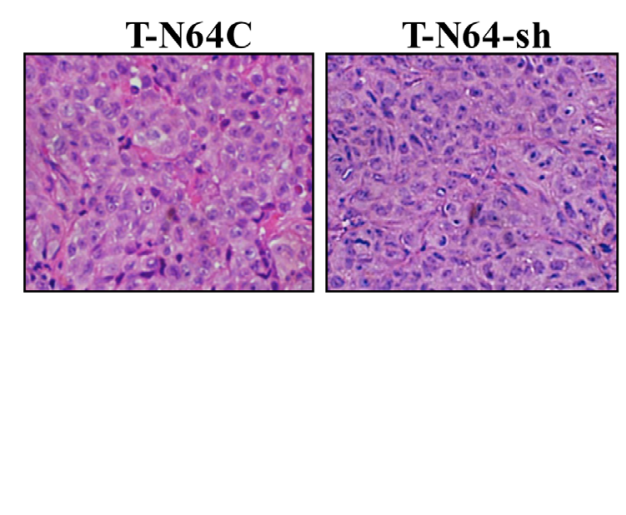

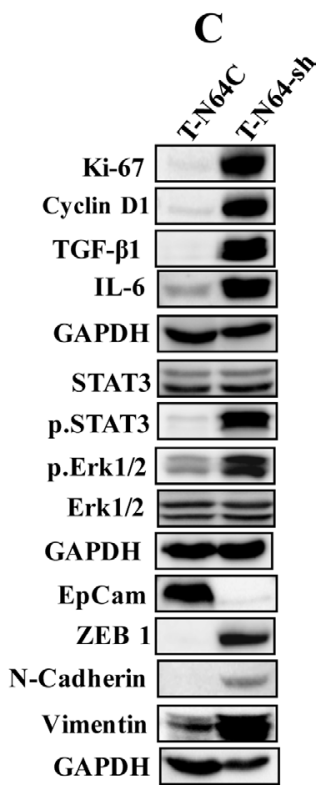

Figure 4: ATR-deficient breast fibroblasts enhance breast orthotopic tumor xenograft formation and growth. Breast cancer orthotopic xenografts were created by co-injecting MDA-MB-231 cells with N64-sh cells (T-N64-sh) or N64C cells (T-N64C) under the nipples of nude mice $(\mathrm{n}=5)$. (A) Graph showing tumor sizes. Error bars represent means \pm S.D. (B) Tissues were subjected to hematoxylin and eosin staining (Envision 40x). (C) Tumors were excised and whole-cell lysates were prepared and protein levels were assessed by immunoblotting using the indicated antibodies. 


\section{Ectopic expression of ATR inactivates breast myofibroblasts}

To further confirm the role of ATR in suppressing the procarcinogenic characteristics of breast stromal fibroblasts, CAF-64 cells were transfected either with a plasmid expressing ATR-ORF (C64-ORF) or the corresponding empty vector (C64C). Figure 5A shows similar number of cells in the various phases of the cell cycle in both cell cultures, indicating that ATR upregulation did not affect the cell cycle distribution. The immunoblotting analysis confirmed the increase in the ATR protein level in C64-ORF cells as compared to C64C cells (Figure 5B). This led to potent p53 up-regulation, and the down-regulation of the SDF-1, $\alpha$-SMA, TGF- $\beta 1$, IL-6 and MMP2 proteins in C64-ORF as compared to the control cells (Figure 5B). Similar results were obtained at the mRNA level (Figure 5C). Moreover, ectopic expression of ATR markedly reduced both the invasion and the migration abilities of breast stromal fibroblasts as compared with control cells (Figure 5D). We have next tested the paracrine effects of these cells on the invasion/ migration abilities of breast cancer cells. To this end, MCF-7 cells were incubated in the presence of SFCM from $\mathrm{C} 64-\mathrm{ORF}$ or $\mathrm{C} 64 \mathrm{C}$, and then their migration/invasion abilities were tested as described above. Figure 5E shows that the expression of ATR in stromal fibroblasts strongly suppressed the migration/invasion abilities of breast cancer cells in a paracrine manner. Together, these results confirm that ATR expression in breast stromal fibroblasts inhibits these cells and suppresses their paracrine procarcinogenic effects.

\section{The effects of ATR-deficiency are p53-dependent}

p53 is a downstream effector of ATR, and we have shown here that p53 expression is modulated in an ATR-dependent manner (Figures 2A and 5B, 5C). Furthermore, p53 plays major roles in repressing breast stromal fibroblasts through regulating the expression of several important genes such as SDF-1 and IL-6 $[13,14]$. Therefore, we decided to investigate the role of p53 in the ATR-deficiency related effects. Therefore, ATR-deficient cells (N64-sh) were transfected with adenovirus-based plasmids expressing TP53-ORF (open reading frame) or a control plasmid. Figure $6 \mathrm{~A}$ shows clear increase in the TP53 mRNA (2.5 fold) in cells expressing TP53-ORF as compared to the controls. On the other hand, mRNA levels of $\alpha$-SMA, SDF-1, MMP-2, IL-6 and TGF- $\beta 1$ were significantly reduced in N64-sh cells expressing TP53-
A

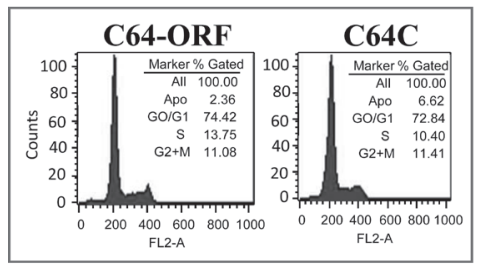

B

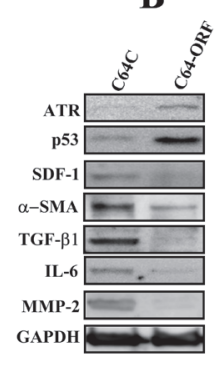

D

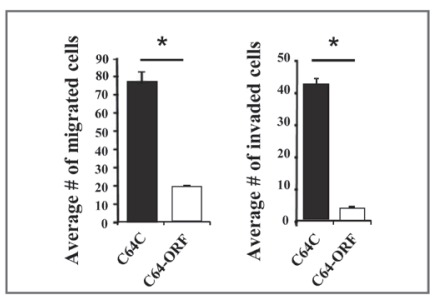

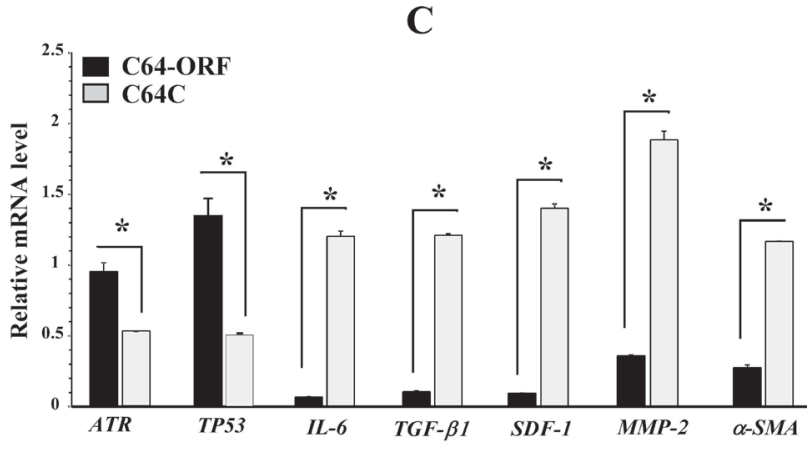

$\mathbf{E}$

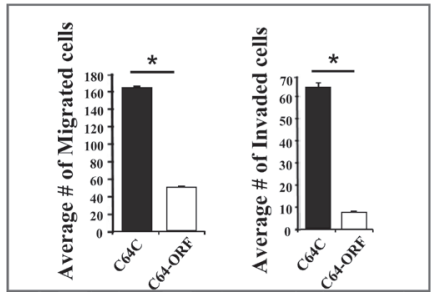

Figure 5: Ectopic expression of ATR suppresses breast myofibroblasts and their paracrine procarcinogenic effects. CAF-64 cells were transfected with ATR-ORF (C64-ORF) or a control plasmid (C64C). (A) cell cycle analysis was performed by flow cytometry. (B) Whole-cell lysates were prepared from the indicated cells and were used for immunoblotting analysis. (C) Total RNA was extracted from the indicated cells, and the mRNA levels of the indicated genes were assessed by qRT-PCR. Error bars represent means \pm SD. ${ }^{*} P<0.01$. (D) C64-ORF and C64C cells $\left(10^{5}\right)$ were seeded in SFM onto the upper compartment of the migration and invasion plates and incubated for $24 \mathrm{~h}$ in the presence of CpM in the lower chamber of 24-well BD BioCoat plates. The number of migrated/invaded cells was represented in histograms. Error bars represent mean $\pm \mathrm{SD}(\mathrm{n}=3)$. ${ }^{*} P<0.0003$. (E) MCF-7 cells were seeded with SFCM from the indicated cells onto the upper compartment of the migration and invasion plates, and then were incubated for $24 \mathrm{~h}$. Error bars represent mean \pm SD. ${ }^{*} P<1.3 \times 10^{-6}$. 
ORF as compared to their respective controls (Figure 6A). Similarly, the secreted levels of IL-6, MMP-2, SDF-1 and TGF- $\beta 1$ were also significantly reduced in N64-sh cells expressing TP53-ORF (Figure 6B). This indicates that ectopic expression of p53 inhibited the effects of ATRdeficiency on the expression/secretion of myofibroblastrelated genes.

Furthermore, TP53 up-regulation significantly reduced the invasiveness and the migratory capabilities of N64-sh cells as compared to their corresponding control cells (Figure 6C). These results indicate that p53 is involved in the ATR-dependent repression of breast stromal fibroblasts.

\section{DISCUSSION}

We have shown here that ATR plays a major role in suppressing BSFs and their pro-carcinogenic effects both in vitro and in vivo. Indeed, specific down-regulation of
ATR increased the proliferation and the migration/invasion abilities of BSFs and also enhanced the expression of the 2 major myofibroblast markers $\alpha$-SMA and SDF-1. In addition, TGF- $\beta 1$, IL-6 and MMP-2 were also up-regulated in ATR-deficient BSFs. These ATR-related effects were inhibited upon ectopic expression of ATR or p53, which indicates that p53 mediates the ATR effects in BSFs. p53 is a well known tumor suppressor gene, which also inhibits BSF through repressing the expression of SDF-1 and IL-6 $[13,14]$. Therefore, p53 has also non-cell-autonomous tumor suppressor function $[15,16]$, and thereby, ATR may also play such role.

The active status of ATR-deficient BSFs was next confirmed by showing that specific down-regulation of ATR enhances the EMT process in breast cancer cells in a paracrine manner. The non-cell-autonomous ATRrelated enhancement of the migration/invasion abilities of breast cancer cells was mediated in an SDF-1/IL-6dependent manner. These 2 cytokines play major roles

\section{A}

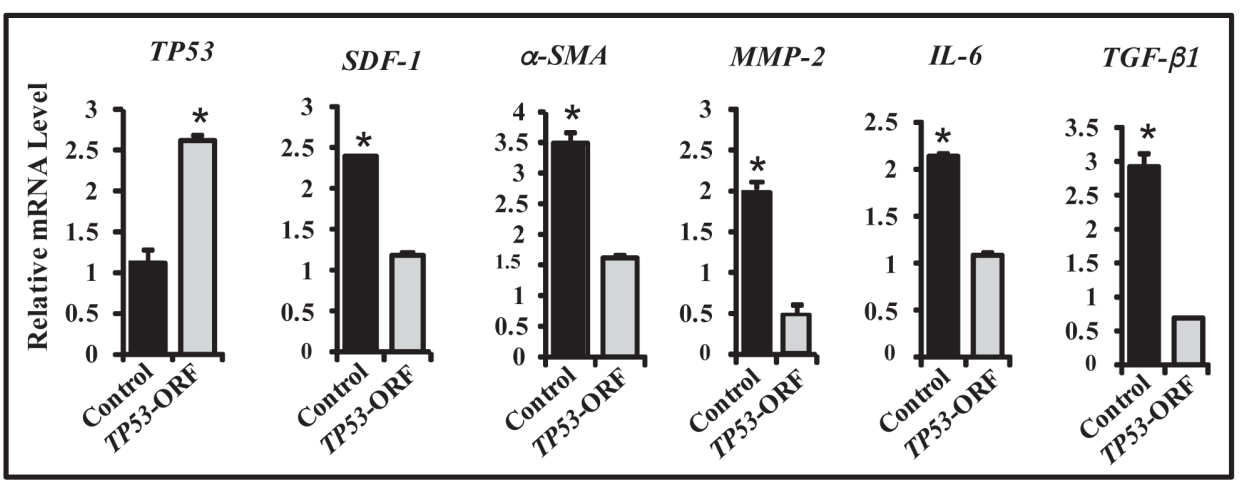

B

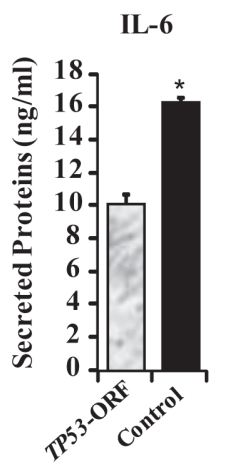

MMP-2

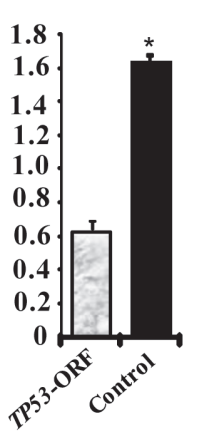

SDF-1

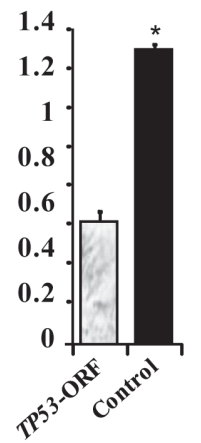

TGF- $\beta 1$

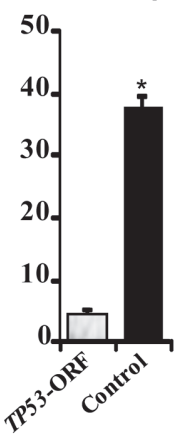

C

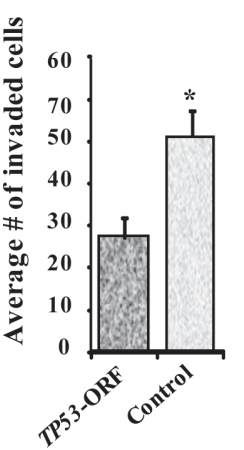

Figure 6: ATR down-regulation activates breast stromal fibroblasts in a p53-dependent manner. N64-sh cells were transfected with a vector bearing TP53-ORF or an empty vector used as control. (A) Total RNA was extracted from the indicated cells, and the mRNA levels of the indicated genes were assessed by qRT-PCR. Error bars represent mean $\pm \mathrm{S} . \mathrm{D}(\mathrm{n}=3)$. ${ }^{*} P<0.017$. (B) SFCM from the indicated cells were collected, and then the secreted levels of the indicated proteins were determined by ELISA. Error bars represent mean \pm S.D $(n=3) .{ }^{*} P<0.0001$. (C) The migration and invasion abilities of the indicated cells were assessed as described in Figure 3F. Error bars represent mean $\pm \mathrm{S} . \mathrm{D}(\mathrm{n}=3)$. ${ }^{*} P<0.009$. 
in the pro-carcinogenic effects of active BSFs [3, 4]. The pro-carcinogenic effects of ATR-deficient breast stromal fibroblasts were corroborated both in vitro using direct coculturing in engineered human breast tissues composed of breast cancer cells and BSFs, and also in vivo through the formation of orthotopic tumors by co-implantation of breast cancer cells and BSFs. In both cases ATRdeficient cells enhanced the growth of breast cancer cells, which was confirmed by showing the expression of high levels of Ki-67 and cyclin D1. In vivo, the presence of ATR-deficient BSFs generated more aggressive tumors wherein STAT3 was highly active and the pro-metastatic genes TGF- $\beta 1$ and IL-6 were highly expressed, and the EMT process was induced. Together, these results indicate that ATR down-regulation in BSFs has potent non-cell-autonomous procarcinogenic effects. Similar effects were previously shown in other tumor suppressor proteins such as p16, PTEN, pRB and CAV-1 $[5,6]$. This indicates that several genes repress the devastating procarcinogenic effects of breast stromal fibroblasts, and that the down-regulation of one of these genes is sufficient to trigger the pro-tumorigenic process through paracrine signaling.

Importantly, we have also shown that ATR is downregulated in 9 out of $12(75 \%)$ CAFs as compared to their corresponding adjacent TCFs isolated from the same breast at adjacent histologically normal tissues. This differential expression suggests that ATR down-regulation in CAFs is due to the presence of these cells close to cancer cells, which perturbs the microenvironment through paracrine signaling. Indeed, we have recently shown that cancer cells activate breast stromal fibroblasts in an IL-6dependent manner [3]. This confirms that ATR is another important target of breast cancer cells in BSFs, and that this protein kinase plays key roles in the carcinoma-stroma cross-talk during breast carcinogenesis.

\section{MATERIALS AND METHODS}

\section{Cells and cell culture}

Breast fibroblast cells were obtained and used as previously described [17]. MCF-7, MDA-MB-231 and MCF-10A cells were purchased from ATCC and were authenticated using short tandem repeat profiling by ATCC, propagated, expanded, and frozen immediately into numerous aliquots after arrival. The revived cells were utilized within 10 to 12 passages and not exceeding a period of 3 months, and were cultured following the instructions of the company. Cells were regularly screened for mycoplasma contamination using MycoAlert Mycoplasma Detection Kits (Lonza). All supplements were obtained from Sigma (Saint Louis, MO, USA) except for antibiotic and antimycotic solutions, which were obtained from Gibco (Grand Island, NY, USA). Cells were maintained at $37^{\circ} \mathrm{C}$ in humidified incubator with $5 \%$
$\mathrm{CO}_{2}$. Human IL-6 recombinant protein (hBA-184) (Santa Cruz, CA). The anti-IL-6 monoclonal antibody (6708.11) was purchased from Sigma-Aldrich, USA. Anti-SDF-1 neutralizing antibody (Human CXCL12/SDF-1 antibody) and IgG (6-101-C-ABS) from R\&D Systems.

\section{Cellular lysate preparation and immunoblotting}

This has been performed as previously described [18]. Antibodies directed against alpha smooth muscle actin ( $\alpha$-SMA), Ki-67, transforming growth factor beta 1 (TGF- $\beta 1$ ), Stromal-derived factor-1 (SDF-1), Twist-1, Vimentin (RV202), MMP-9, (ab38898), ATR (ab54793), N-cadherin and interleukin-6 (IL-6) were purchased from Abcam (Cambridge, MA); STAT3, pSTAT3Tyr705 (D3A7), Snail (C15D3), E-cadherin (24E10), EpCAM (UV1D9), JAK-2 (D2E12) and phospho-JAK-2 (TYR1007/1008), CyclinD1 (2922), Akt, phospho-Akt (193H12), MMP-2 (4022), ERK1/2 (137F5) and phosphoERK1/2 from Cell Signaling (Danvers, MA); ZEB-1 (4C4) from Abnova (Taipei, Taiwan); p21 (F-5), p53 (DO-1) and Glyceraldehydes-3-phosphate dehydrogenase (GAPDH, FL-335) were purchased from Santa Cruz (Santa Cruz, CA).

\section{RNA purification, RT-PCR and qRT-PCR}

Total RNA was purified using the TRI reagent (Sigma) according to the manufacturer's instructions, and was treated with RNase-free DNase before cDNA synthesis using the RT-PCR Kit (Clontech, USA). cDNA was amplified using the Platinum ${ }^{\circledR}$ Taq DNA Polymerase (Invitrogen). The $\mathrm{RT}^{2}$ Real-Time ${ }^{\mathrm{TM}}$ SYBR Green qPCR mastermix (Roche, Germany) was used and the amplifications were performed utilizing the light cycler 480 (Roche, Germany). The melting-curve data were collected to check PCR specificity, and the amount of PCR products was measured by threshold cycle $(\mathrm{Ct})$ values and the relative ratio of specific genes to $G A P D H$ for each sample was then calculated. The respective primers are:

TP53: 5'-CAGTCTACCTCCCGCCATAA-3' and

5'- CCACAACAAAACACCAGTGC-3'

MMP-2: 5'-CATGTCGCCCCTAAAACAGA-3' and 5'-CCATCAAACGGGTATCCATC-3'

GAPDH: 5'-GAGTCCACTGGCGTCTTC-3' and

5'-GGGGTGCTAAGCAGTTGGT-3'

$S D F-1$ : 5'-TAGTCAAGTGCGTCCACGA-3' and

5'-GGACACACCACAGCACAAAC-3'

$\alpha-S M A: 5^{\prime}$-CCGACCGAATGCAGAAGGA-3' and

5'-ACAGAGTATTTGCGCTCCGAA-3'

TGF- $\beta 1$ : 5'-TGTGTGCTGAAGCCATCGTTG-3' and 5'-CCGGCTTGTCTGAAAAGGTCA-3'

IL-6: 5'-GACAAAGCCAGAGTCCTTCAGAGA-3' and 5'-CTAGGTTTGCCGAGTAGATCT-3'

ATR: 5'-GTCATATACACTCCCTTTTCTTTA-3' and 5'-GTCATATACACTCCCTTTTCTTTA-3' 


\section{ATR-shRNA transfection}

ATR-shRNA (KHD1318) expressed in sure silencing shRNA plasmid and the corresponding control plasmid were obtained from GenScript Corporation, and were used to carry out transfection using human dermal fibroblast nucleofector 2000 transfection kit (Invitrogen) following the protocol recommended by the manufacturer.

\section{Viral infection}

Lentivirus based vectors bearing TP53-ORF as well as the respective control (Origene) were used to prepare the corresponding lentiviral supernatants from 293FT cells. Lentiviral supernatants were collected 48 $\mathrm{h}$ post-transfection, filtered and used for infection. Cells were infected for $48 \mathrm{~h}$, and then media were replaced with complete media and cells were grown for 3 days.

\section{ATR-ORF}

Lentivirus based vectors bearing ATR-ORF, (pLentiC-Myc-DDK) and the corresponding control were used to carry out transfection using human dermal fibroblast nucleofector 2000 transfection kit (Invitrogen) following the manufacturer's recommendations. After ten days of transfection, cells were tagged using OriGene anti-DDK antibody (TA50011) and sorted with BD FACSAria cell sorting system.

\section{ELISA assays}

Supernatants from $24 \mathrm{~h}$ fibroblast cell cultures were harvested and ELISA was performed according to the manufacturer's instructions (R\&D Systems). The OD was used at 450-nm on a standard ELISA plate-reader. These experiments were performed in triplicates.

\section{Chemotaxis and invasion assays}

The 24-well BD BioCoat Matrigel Invasion chambers were used as per the manufacturer guideline (BD Bioscience). 2-4 x 105 cells were added to the upper wells separated by an 8 micron pore size PET membrane with a thin layer of matrigel basement membrane matrix (for invasion) or without (for migration). The membranes were stained with Diff Quick stain (Fisher Scientific) after removing the non-migrated cells from the top of the membrane with Q-tips. After airdrying, the membranes were cut and mounted on slides with oil, and cells that had migrated to the underside of the filter were counted using light microscope (Zeiss Axio Observer) in five randomly selected fields (magnification; 40x). Each assay was performed in triplicate.

\section{Cell proliferation}

This assay was performed in a real-time and labelfree manner using the xCELLigence RTCA technology (Roche, Germany) that measures impedance changes in a meshwork of interdigitated gold microelectrodes located at the bottom well (E-plate). Exponentially growing cells $\left(2 \times 10^{4}\right)$ were seeded in E-plate with complete medium as per manufacturer's instruction. All data were recorded and analyzed by the RTCA software. Cell Index (CI) was used to measure the change in the electrical impedance divided by the background value to represent cell status. Each assay was performed in triplicate.

\section{Conditioned media}

Cells were cultured in medium without serum for 24 $\mathrm{h}$, and then media were collected and briefly centrifuged. The resulting supernatants were used either immediately or were frozen at $-80^{\circ} \mathrm{C}$ until needed.

\section{Immunohistochemistry staining on FFPE tissues}

Immunohistochemistry for ATR was done on formalin-fixed, paraffin-embedded tissues using anti-ATR antibody from abcam (ab54793) at a dilution of 1:500 and tissues were stained using automated staining platform (Ventana). Envision + polymer (ready to use; Dako) was used as a secondary antibody. Color was developed with 3,3'-diaminobenzidine (DAB) and instant hematoxylin (Shandon) was used for counterstaining.

\section{Orthotopic tumor xenografts}

Animal experiments were approved by the KFSH\&RC institutional Animal Care and Use Committee (ACUC) and were conducted according to relevant national and international guidelines. 10 female nude mice were randomized into 2 groups and breast cancer orthotopic xenografts were created by coimplantation of MDA-MB-231 cells $\left(2 \times 10^{6}\right)$ with N64-sh, or N64C cells $\left(2 \times 10^{6}\right)$ under the nipple of each mouse. Tumor size was measured with a caliper using the following formula (Length X Width X Height).

\section{Statistical analysis}

Statistical analysis was performed by student's t-test and $P$ values of 0.05 and less were considered as statistically significant.

\section{ACKNOWLEDGMENTS}

We would like to thank Dr. Huda Al-Khalaf for her help and suggestions. We are also very thankful to the $\mathrm{KFSH} \& \mathrm{RC}$ administration for the continuous support. 


\section{CONFLICTS OF INTEREST}

No competing interests are declared by any of the authors.

\section{REFERENCES}

1. Kalluri R. The biology and function of fibroblasts in cancer. Nat Rev Cancer. 2016; 16:582-598.

2. Gascard P, Tlsty TD. Carcinoma-associated fibroblasts: orchestrating the composition of malignancy. Genes Dev. 2016; 30:1002-1019.

3. Al-Ansari MM, Aboussekhra A. miR-146b-5p mediates p16-dependent repression of IL-6 and suppresses paracrine procarcinogenic effects of breast stromal fibroblasts. Oncotarget. 2015; 6:30006-30016. https://doi.org/10.18632/ oncotarget.4933.

4. Orimo A, Gupta PB, Sgroi DC, Arenzana-Seisdedos F, Delaunay T, Naeem R, Carey VJ, Richardson AL, Weinberg

RA. Stromal fibroblasts present in invasive human breast carcinomas promote tumor growth and angiogenesis through elevated SDF-1/CXCL12 secretion. Cell. 2005; 121:335-348.

5. Aboussekhra A. Role of cancer-associated fibroblasts in breast cancer development and prognosis. Int J Dev Biol. 2011; 55:841-849.

6. Al-Ansari MM, Hendrayani SF, Shehata AI, Aboussekhra A. p16(INK4A) Represses the paracrine tumor-promoting effects of breast stromal fibroblasts. Oncogene. 2013; 32:2356-2364.

7. Weber AM, Ryan AJ. ATM and ATR as therapeutic targets in cancer. Pharmacol Ther. 2015; 149:124-138.

8. Perou CM, Sørlie T, Eisen MB, van de Rijn M, Jeffrey SS, Rees CA, Pollack JR, Ross DT, Johnsen H, Akslen LA, Fluge O, Pergamenschikov A, Williams C, et al. Molecular portraits of human breast tumours. Nature. 2000; 406:747-752.

9. Zhang SY, Liu SC, Al-Saleem LF, Holloran D, Babb J, Guo X, Klein-Szanto AJ. E2F-1: a proliferative marker of breast neoplasia. Cancer Epidemiol Biomarkers Prev. 2000; 9:395-401

10. Alderton GK, Joenje H, Varon R, Borglum AD, Jeggo PA, O'Driscoll M. Seckel syndrome exhibits cellular features demonstrating defects in the ATR-signalling pathway. Hum Mol Genet. 2004; 13:3127-3138.

11. O’Driscoll M, Ruiz-Perez VL, Woods CG, Jeggo PA, Goodship JA. A splicing mutation affecting expression of ataxia-telangiectasia and Rad3-related protein (ATR) results in Seckel syndrome. Nat Genet. 2003; 33:497-501.

12. Heikkinen K, Mansikka V, Karppinen SM, Rapakko K, Winqvist R. Mutation analysis of the ATR gene in breast and ovarian cancer families. Breast Cancer Res. 2005; 7:R495-501.

13. Addadi Y, Moskovits N, Granot D, Lozano G, Carmi Y, Apte RN, Neeman M, Oren M. p53 status in stromal fibroblasts modulates tumor growth in an SDF1-dependent manner. Cancer Res. 2010; 70:9650-9658.

14. Margulies L, Sehgal PB. Modulation of the human interleukin-6 promoter (IL-6) and transcription factor C/ EBP beta (NF-IL6) activity by p53 species. J Biol Chem. 1993; 268:15096-15100.

15. Kiaris H, Chatzistamou I, Trimis G, FrangouPlemmenou M, Pafiti-Kondi A, Kalofoutis A. Evidence for nonautonomous effect of p53 tumor suppressor in carcinogenesis. Cancer Res. 2005; 65:1627-1630.

16. Lujambio A, Akkari L, Simon J, Grace D, Tschaharganeh DF, Bolden JE, Zhao Z, Thapar V, Joyce JA, Krizhanovsky V, Lowe SW. Non-cell-autonomous tumor suppression by p53. Cell. 2013; 153:449-460.

17. Hawsawi NM, Ghebeh H, Hendrayani SF, Tulbah A, Al-Eid M, Al-Tweigeri T, Ajarim D, Alaiya A, Dermime S, Aboussekhra A. Breast carcinoma-associated fibroblasts and their counterparts display neoplastic-specific changes. Cancer Res. 2008; 68:2717-2725.

18. Al-Mohanna MA, Al-Khalaf HH, Al-Yousef $\mathrm{N}$, Aboussekhra A. The p16INK4a tumor suppressor controls p21WAF1 induction in response to ultraviolet light. Nucleic Acids Res. 2007; 35:223-233 